\title{
Formation of a three-dimensional plasma boundary after decay of the plasma response to resonant magnetic perturbation fields
}

O. Schmitz

T. E. Evans

M. E. Fenstermacher

M. J. Lanctot

Saskia Mordijck

William \& Mary, smordijck@wm.edu

Follow this and additional works at: https://scholarworks.wm.edu/aspubs

Part of the Physics Commons

\section{Recommended Citation}

Schmitz, O.; Evans, T. E.; Fenstermacher, M. E.; Lanctot, M. J.; and Mordijck, Saskia, Formation of a threedimensional plasma boundary after decay of the plasma response to resonant magnetic perturbation fields (2014). Nuclear Fusion, 54(1). https://www.doi.org/10.1088/0029-5515/54/1/012001

This Article is brought to you for free and open access by the Arts and Sciences at W\&M ScholarWorks. It has been accepted for inclusion in Arts \& Sciences Articles by an authorized administrator of W\&M ScholarWorks. For more information, please contact scholarworks@wm.edu. 


\title{
Formation of a three-dimensional plasma boundary after decay of the plasma response to resonant magnetic perturbation fields
}

\author{
O. Schmitz ${ }^{1}$, T.E. Evans ${ }^{2}$, M.E. Fenstermacher ${ }^{3}$, M.J. Lanctot ${ }^{2}$, \\ C.L. Lasnier ${ }^{3}$, S. Mordijck ${ }^{4}$, R.A. Moyer ${ }^{5}$, H. Reimerdes ${ }^{6}$ and \\ the DIII-D Team
}

${ }^{1}$ Institut für Energieforschung-Plasmaphysik, Forschungszentrum Jülich $\mathrm{GmbH}$, Association EURATOM-FZJ, Trilateral Euregio Cluster, 52425 Jüelich, Germany

${ }^{2}$ General Atomics, PO Box 85608, San Diego, CA 92186-5608, USA

${ }^{3}$ Lawrence Livermore National Laboratory, Livermore, CA 94550, USA

${ }^{4}$ College of William and Mary, Williamsburg, VA 21387, USA

${ }^{5}$ University of California, San Diego, La Jolla, CA 92093-0417, USA

${ }^{6}$ Ecole Polytechnique Fèdèrale de Lausanne (EPFL), CRPP, Association

Euratom-Confèdèration Suisse, Lausanne, Switzerland

E-mail: o.schmitz@fz-juelich.de

Received 18 July 2013, revised 19 November 2013

Accepted for publication 28 November 2013

Published 17 December 2013

\begin{abstract}
First time experimental evidence is presented for a direct link between the decay of a $n=3$ plasma response and the formation of a three-dimensional (3D) plasma boundary. We inspect a lower single-null L-mode plasma which first reacts at sufficiently high rotation with an ideal resonant screening response to an external toroidal mode number $n=3$ resonant magnetic perturbation field. Decay of this response due to reduced bulk plasma rotation changes the plasma state considerably. Signatures such as density pump out and a spin up of the edge rotation - which are usually connected to formation of a stochastic boundary-are detected. Coincident, striation of the divertor single ionized carbon emission and a 3D emission structure in double ionized carbon at the separatrix is seen. The striated $C_{\text {II }}$ pattern follows in this stage the perturbed magnetic footprint modelled without a plasma response (vacuum approach). This provides for the first time substantial experimental evidence, that a 3D plasma boundary with direct impact on the divertor particle flux pattern is formed as soon as the internal plasma response decays. The resulting divertor structure follows the vacuum modelled magnetic field topology. However, the inward extension of the perturbed boundary layer can still not directly be determined from these measurements.
\end{abstract}

Keywords: plasma wall interaction, stochastic boundary plasma, plasma response, resonant magnetic perturbation fields, ELM suppression

(Some figures may appear in colour only in the online journal)

\section{Introduction}

A key requirement for operation of high temperature plasmas in the high confinement mode (H-mode) regime [1] is the control of large, type-I edge localized modes (ELMs). These edge instabilities endanger the wall integrity of future fusion experiments such as ITER with a potential degradation of the plasma performance [2]. One promising method to suppress such type-I ELMs is the use of external magnetic perturbation fields. This was demonstrated at DIII-D using small amplitude, toroidal mode number $n=3$ edge resonant magnetic perturbation (RMP) fields for a variety of plasma shapes and collisionalities [3,4] including, high triangularity $(\bar{\delta})$, ITER similar shape (ISS) plasmas at low electron pedestal collisionality $v_{\mathrm{e}}^{*}[5]$. Subsequently ELM control by external magnetic perturbations was investigated at Asdex-Upgrade [6], JET [7], KSTAR [8], MAST [9, 10] and NSTX [11]. For the extrapolation of this method to future devices, the generic 
mechanisms of the method have to be understood. One high priority question is, what is the plasma response to the external RMP fields and the resulting magnetic topology in the plasma edge. Both determine the plasma transport and therefore the ELM stability with RMP.

The working hypothesis for RMP ELM suppression was proposed in $[3,12]$, employing enhanced radial transport by introducing a stochastic layer due to the external RMP field. This enhanced transport can control the edge pressure gradients and is capable of stabilizing the ballooning part of the peelingballooning stability type-I ELM. In [13,14] enhancement of thermal transport and a resonant (i.e. $q_{95}$ dependent) pressure reduction was discussed which is also compatible with some level of edge stochastization. However, the confirmation of this stochastic edge layer, its topology and in particular its radial extension is still pending. One natural approach to look for experimental signatures of this stochastic boundary is to inspect the very plasma edge and the interaction with the first wall, in particular with the divertor target surfaces. This was successfully applied at TEXTOR to investigate the impact of shielded RMP fields on the target ion fluxes [15]. At DIII-D a striation of the divertor heat and particle fluxes was observed during ELM suppression with a clear dependence of the heat and particle flux magnitudes and geometry on the plasma collisionality $v_{\mathrm{e}}^{*}$ and shape [16-18]. This striation is caused by the interaction of the external RMP field with the stable and unstable manifolds of the separatrix. The lobes of these manifolds get decomposed under the influence of the RMP field and form at the target surface a helical, three-dimensional (3D) magnetic footprint [17-20]. Recently these separatrix lobes have been directly imaged at DIII-D [21] and MAST [22]. At other devices applying external magnetic perturbation fields, a similar striation of divertor fluxes was measured under specific conditions. At NSTX the heat and particle fluxes split under the influence of magnetic perturbation fields when $n=3$ fields are used to trigger ELMs [23]. At JET and MAST, a striation is so far seen only during low power L-mode experiments [24]. These observations point out in summary the urgent need to understand to what extent the plasma response and this strike line striation as a signature for a 3D shape of the plasma boundary are related. In this paper we understand the striated divertor footprint pattern as a signature for the $3 \mathrm{D}$, helical separatrix structure and call this a 3D plasma boundary. When RMP fields are applied, residual resonant components which are not fully shielded out will in most cases result in a residual 3D boundary structure with good flux surfaces. However, when the field actually penetrates, flux surfaces are broken up and due to the perturbation of the separatrix a 3D perturbed edge layer consisting out of open field lines is formed. In this paper we present for the first time direct experimental evidence for a link between the decay of a $n=3$ magnetic plasma response measured and the formation of the $3 \mathrm{D}$ plasma boundary in this regard.

\section{Measurement of the decaying $n=3$ plasma response}

We inspect in this study DIII-D L-mode plasma discharge \#142614 in lower single-null (LSN) diverted shape. The typical plasma parameters of this discharge are plasma current

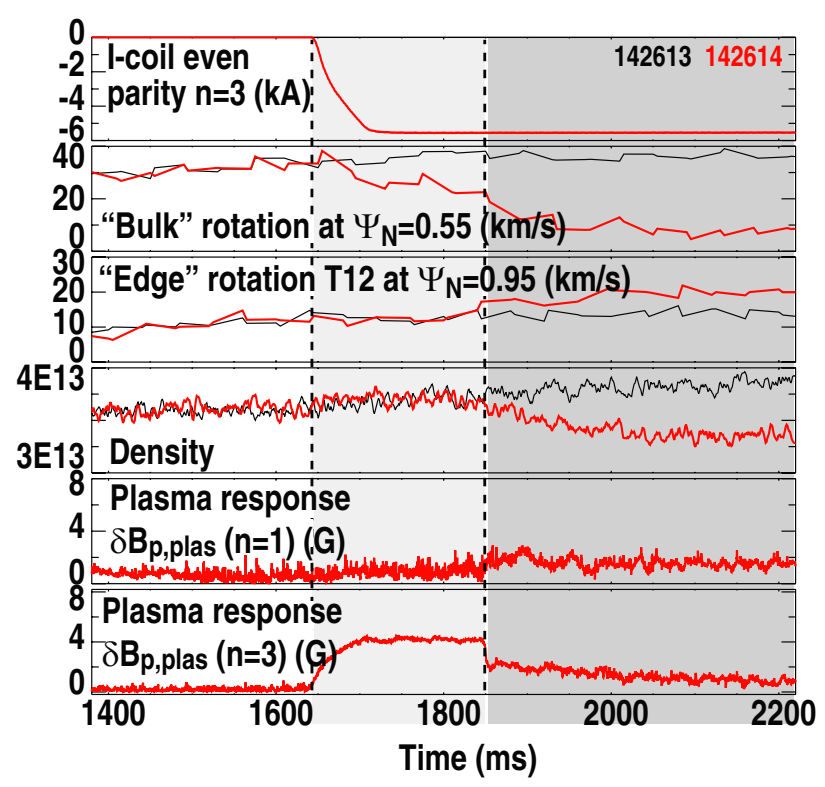

Figure 1. Time traces of plasma discharge during $n=3$ RMP application.

$I_{\mathrm{P}}=1.4 \mathrm{MA}$, toroidal magnetic field $B_{\mathrm{T}}=-2.0 \mathrm{~T}$, total heating power $P_{\mathrm{H}}=2.51 \mathrm{MW}$, mean averaged triangularity $\bar{\delta}=0.52$, central electron density $n_{\mathrm{ec}}=4.0 \times 10^{19} \mathrm{~m}^{-3}$, $\beta_{\mathrm{N}}=0.5$. Figure 1 shows (from top to bottom) the time traces of I-coil RMP current $I_{\mathrm{I}-\text { coil }}(\mathrm{kA})$, central plasma rotation $v_{\text {tor c }}\left(\mathrm{km} \mathrm{s}^{-1}\right)$ (channel T6 at $\left.\Psi_{\mathrm{N}}=0.55\right)$, edge toroidal rotation $v_{\text {tor e }}\left(\mathrm{km} \mathrm{s}^{-1}\right)\left(\right.$ channel T12 at $\left.\Psi_{\mathrm{N}}=0.95\right)$, central plasma density $n_{\mathrm{ec}}\left(\mathrm{cm}^{-3}\right), n=1$ poloidal component of the plasma response $\delta B_{\mathrm{p} \text {,plasma }}(n=1) \mathrm{G}$ and $n=3$ poloidal component of the plasma response $\delta B_{\text {p,plasma }}(n=3) \mathrm{G}$. The $n=3$ dominated RMP field with a spectrum typically applied for RMP ELM suppression at an I-coil current of $I_{\mathrm{I}-\text { coil }}=5.5 \mathrm{kA}$ is applied from $t=1600-4400 \mathrm{~ms}$. After energizing the RMP field, a $n=3$ poloidal magnetic field as plasma response amplitude $B_{\mathrm{p} \text {,plasma }}=4.2 \mathrm{G}$ is measured. The response is proportional to the I-coil current and has no delay. The plasma response measurement is obtained as resulting magnetic plasma response field of the $n=3$ mode component measured by Mirnov coils at the vessel wall [25]. The plasma response was calculated with the MARS-F code using an ideal MHD plasma model [26]. Ideal MHD assumes perfect screening of the resonant external field. While finite resistivity should lead to a decay of these screening currents, plasma rotation is thought to maintain a high level of screening. [28] The magnetic field amplitudes are shown in figure 2 as poloidal harmonics of the RMP spectrum applied for toroidal mode number $n=3$. We compare the spectral amplitudes of the vacuum field (figure $2(a)$ ) and the left handed helicity spectrum modelled with MARS-F (figure $2(b)$ ). The dashed line marks the trace of rational surfaces satisfying $m=n q$ with $m$ being the poloidal and $n$ being the toroidal mode number. Table 1 shows a quantitative comparison between the experimental measurement by the external magnetic sensors at DIII-D [25] and the MARS-F modelling. A fair agreement in the total amplitudes and the toroidal phase of the plasma response field is found. The calculated plasma response is identified as resulting from sheet currents on the resonant 

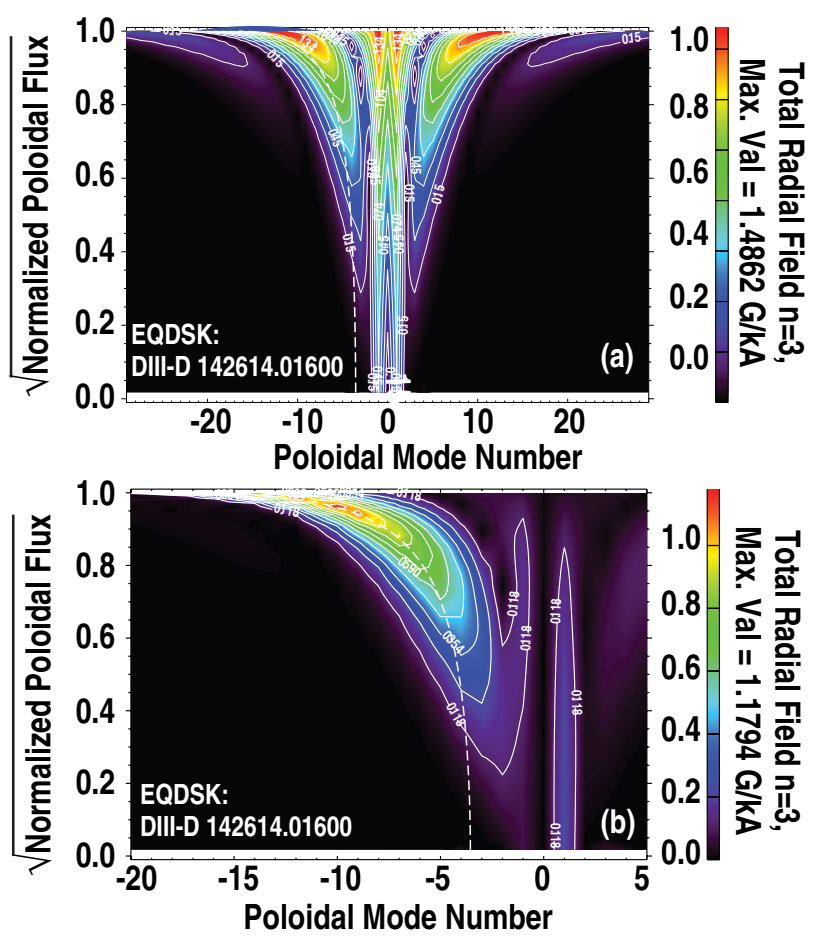

Figure 2. Vacuum spectral perturbation amplitudes of the $n=3$ (a) components and (b) MARS-F plasma response modelling.

Table 1. Comparison of plasma response measurement and modelling for \#142614.

\begin{tabular}{lll}
\hline & $\begin{array}{l}\text { Amplitude } \\
B_{\text {p,plasma }}\left(\mathrm{G} \mathrm{kA}^{-1}\right)\end{array}$ & $\begin{array}{l}\text { Toroidal } \\
\text { phase } \Phi\left({ }^{\circ}\right)\end{array}$ \\
\hline Experiment & $0.764 \pm 0.042$ & $+95.0 \pm 3.6$ \\
MARS-F & 0.528 & +84.8 \\
\hline
\end{tabular}

surfaces, which act to screen externally applied pitch-resonant fields (i.e. $m=n q$ ). Excitation of poloidal harmonics associated with the $n=1$ kink mode, as observed in high beta plasmas, was not seen in the calculated mode structure for this low beta equilibrium [27]. The toroidal phase is the phase shift of the measured/modelled resonant field amplitudes relative to the radial magnetic field structure at mid plane. The phase of $90^{\circ}$ as measured and modelled means in this respect that a harmonic field is generated in the plasma with a significant toroidal shift against the external field. As the separatrix determines the structure of the measured particle fluxes on the divertor, the relative phase shift is capable yielding a destructive interference of externally applied and internally generated (response) fields at the separatrix such that only reduced radial components are seen by the separatrix during the plasma response phase. This process is capable to reduce the width and radial extension of the overlap region of the invariant manifolds.

The plasma response decays rapidly within $\Delta t_{\text {decay }}=$ $12 \mathrm{~ms}$ at $t=1830 \mathrm{~ms}$. This decay of the plasma response field is potentially caused by the decrease of the core plasma rotation which is observed when the RMP field is applied. This is seen in the bulk rotation time trace in figure 1 and documented in detail in the profiles of toroidal rotation shown in figure 3. Here, four toroidal rotation profiles are shown starting at the

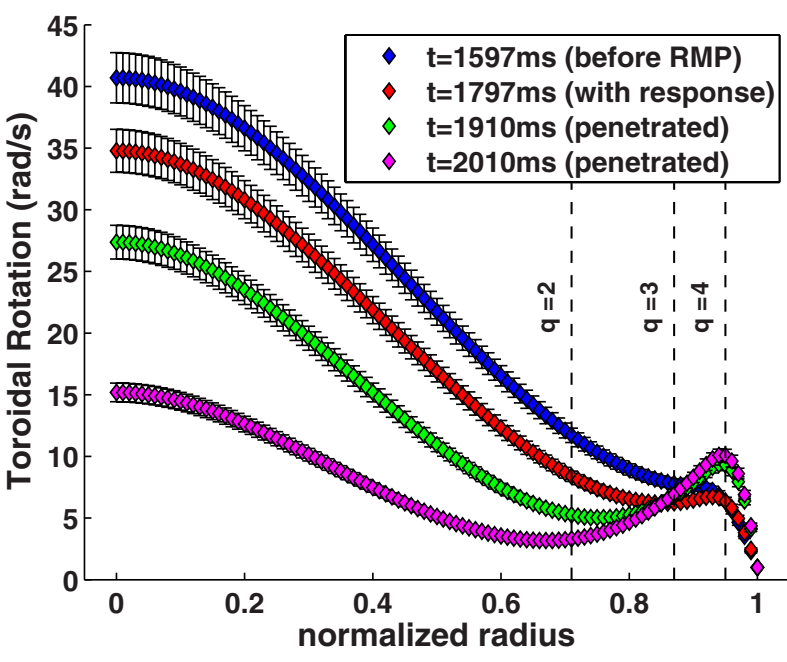

Figure 3. Profiles of toroidal rotation during the RMP application and penetration phase.

discharge time without RMP field applied, during the screened phase and two more profiles during the penetrated state when the plasma response has decayed. A decrease of the core rotation is measured as soon as the RMP field is applied and continues to decrease. The profiles show the radial evolution of this process. After RMP field is applied, the rotation profile in general is dragged down and as soon as the field penetrates, the rotation braking in the core increases with a simultaneous spin up the edge rotation. Following [28], the process of rotational breaking can yield breakdown of the screening when a bifurcation in the rotation occurs at the resonant surface allowing field penetration. The $q=2$ surface is the resonant surface with the strongest braking and hence penetration to this surface followed by locking of the magnetic island and further plasma braking as observed is suggested. However, at the same time a significant spin up at the plasma edge is seen, once the measured plasma response decayed. The resulting penetrated plasma state is characterized by density pump out as well as this rotation spin up in the plasma edge. Both are established signatures during RMP application and usually connected to the formation of a stochastic boundary. It is important to note that the density pump out only sets in when the screening field has decayed. This is consistent with the fact that manipulation of the edge carbon and hydrogen emission discussed later occurs only after the field penetrated. Until then the plasma response avoids penetration of the external field and hence the strong deformation of the separatrix and formation of a open, perturbed edge layer by overlapping separatrix manifolds as observed in the penetrated state does not occur. Both plasma effects, i.e. density pump out and edge rotation spin up, are not completely understood. A more detailed analysis of a similar sequence of RMP L-mode discharges can be found in [29]. Analysis of circular shaped limiter discharges at TEXT, Tore-Supra and TEXTOR suggest that the particle pump out is potentially caused by enhanced radial transport in combination with a change in the neutral source distribution and fuelling $[19,30,31]$. The edge rotation spin up is compatible with a fast electron loss along the perturbed open field lines in a stochastic edge [32] balanced by a radial outward ion current preserving ambipolarity. This ion current can drive rotation by $j \times B$ forces [33-35]. The experimental observations discussed 


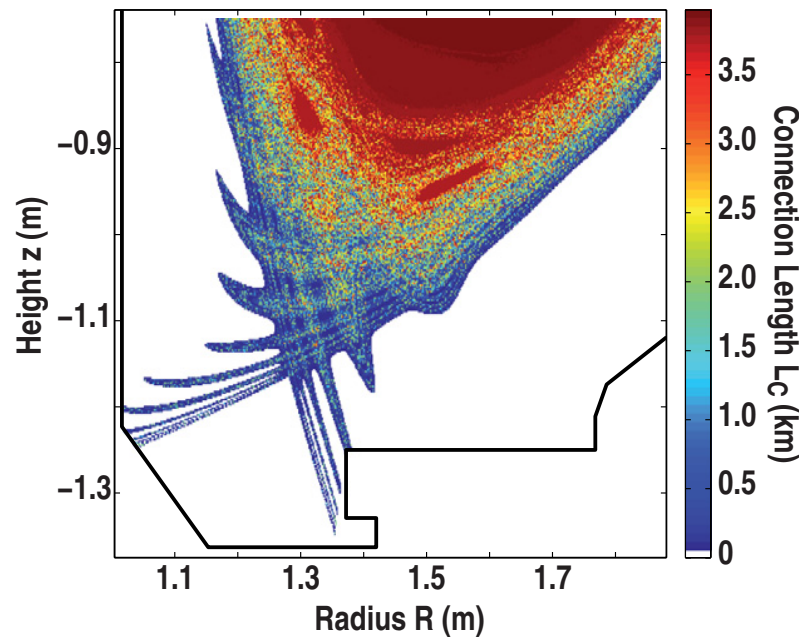

Figure 4. Magnetic topology of the perturbed X-point from vacuum field line tracing.

therefore provide experimental evidence for a decaying ideal plasma response connected to signatures which are considered as signatures for formation of a stochastic boundary layer. The first step establishing this connection for the discharge under discussion is now to inspect the related changes at very edge of the plasma.

\section{Formation of a 3D boundary after decay of the plasma response}

One generic signature of such a stochastic boundary-even in case the field does not penetrate as predicted by the vacuum assumption - is the formation of a 3D plasma boundary-i.e. the helical deformation of the separatrix as introduced and shown in figure 4. Here the magnetic field line topology is depicted displaying the wall to wall connection length of the magnetic field lines. The lobes of the separatrix manifolds are clearly seen and, as discussed in $[17,20]$, they form the outermost, helical and 3D plasma boundary. The interaction of the RMP field with the separatrix is not a resonant coupling process (in contrast to the formation of magnetic islands inside of the plasma) but the 3D shape of the boundary is determined by the sum of all radial perturbations at the separatrix. This means that the decomposition of the separatrix manifolds depends on the balance of the radial components of the external RMP spectrum at the separatrix with all additional radial magnetic fields, such as those generated by internal response currents. If they have-as measured and modelled by MARS-F- a phase relative to the vacuum case, the radial fields can destructively interfere at the separatrix with the result that the separatrix manifolds only experience a small resulting perturbation field. This concept points out that measurements of the separatrix intersection with the divertorsuch as divertor flux distributions - are capable of providing evidence if plasma response currents exist. Accordingly we expect a strong change of the magnetic and plasma topology going from the phase with ideal resonant screening to the penetrated case.

To inspect this going from $(a)$ the stage without RMP application, $(b)$ to the time when the $n=3$ plasma response is
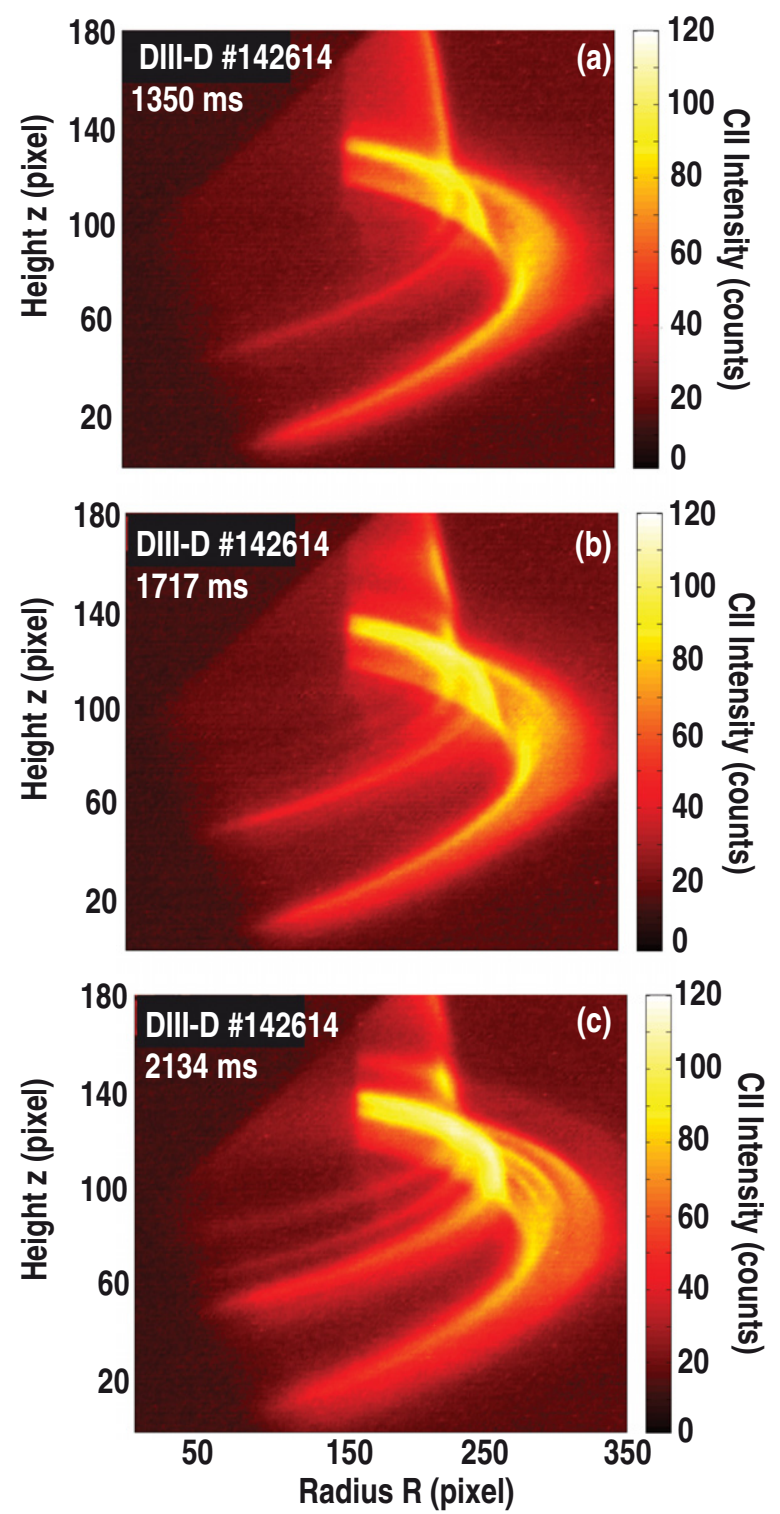

Figure 5. Emission of double ionized carbon C III@465 nm during (a) no RMP field application, (b) with RMP field and $n=3$ plasma response and $(c)$ after decay of the plasma response.

present and $(c)$ to the case where the response decayed, we first look with a tangential observing camera system into the lower divertor [36]. Figures 5(a)-(c) show, for the above sequence, the line emission of double ionized carbon (C III @ $465 \mathrm{~nm}$ ) in the lower divertor X-point region. Before RMP application, there is no $3 \mathrm{D}$ structure seen at the separatrix. The emission pattern looks rather symmetric with divertor legs clearly visible as straight lines crossing at the X-point. As soon as the RMP field is applied, but shielded by the ideal resonant screening, a slight change of the $\mathrm{C}$ III emission pattern is seen indicating a deformation of the boundary, however, without a clear 3D boundary evolution. After decay of the plasma response and connected to the density pump out and edge rotation spin up, a pronounced $3 \mathrm{D}$ separatrix structure is seen in the $\mathrm{C}_{\mathrm{III}}$ emission with a striation of the divertor strike line towards the target surfaces. However, this low power L-mode populates the $\mathrm{C}_{\text {III }}$ state only at considerable distances from the target. Therefore we need to investigate the lowest carbon ionized 


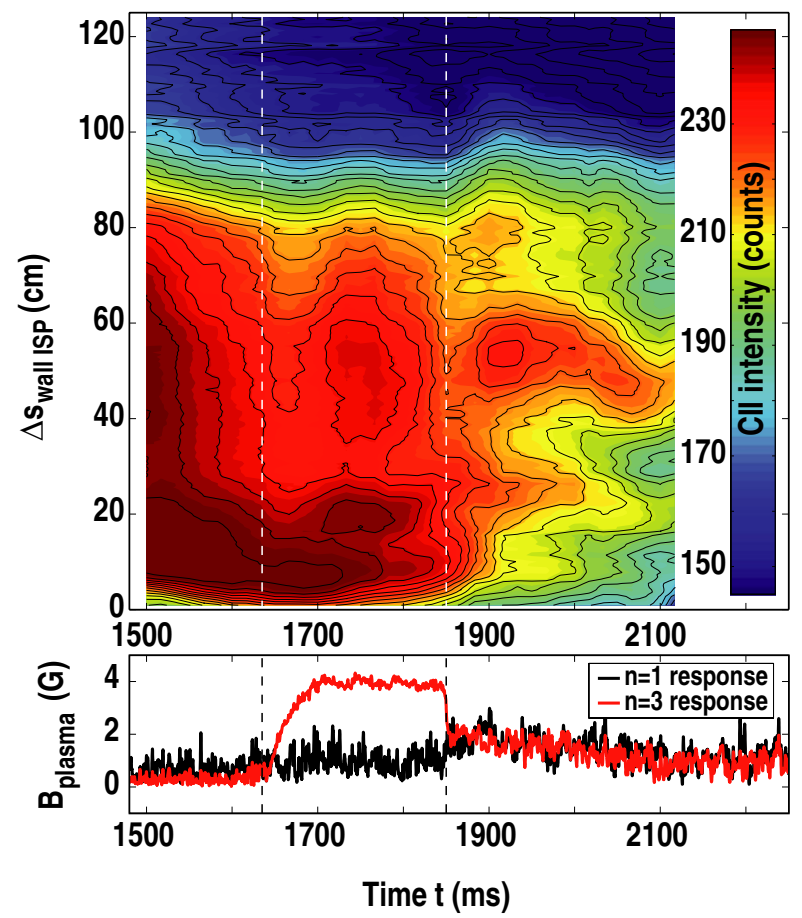

Figure 6. $\mathrm{C}$ II emission at the inner divertor leg during decay of the plasma response.

state, i.e. C II. For this we utilize a camera which observes the inner strike point (ISP) with a perpendicular view down into the divertor from top of DIII-D (see [17,18] for a detailed description of the setup). Figure 6 shows the time evolution of the $\mathrm{C}_{\mathrm{II}}\left(\Delta s_{\mathrm{wall}}\right)$ profile, where $\Delta s_{\text {wall }}$ is the distance from the separatrix at the inner leg divertor target relative to the ISP position. The lower part of the figure shows the $n=3$ and $n=1$ plasma response fields on the same time scale. Before the RMP field is applied the $\mathrm{C} \operatorname{II}\left(\Delta s_{\text {wall }}\right)$ does not show any striation. As soon as the RMP field is applied, a reduction in the intensity is seen with a marginal modulation along the profile. As soon as the plasma response is decayed the typical strike line striation is measured indicating that at this stage the 3D boundary layer is established similar to the observations during ELM suppression at DIII-D. To compare this in more detail to the vacuum magnetic topology, figure 7 shows a comparison of the $\mathrm{C} \operatorname{II}\left(\Delta s_{\text {wall }}\right)$ profiles at the different time steps with the magnetic footprint profile at the measurement location calculated by vacuum field line tracing. The profile of the deepest radial point reached in terms of normalized toroidal flux $\Psi_{\mathrm{N}}$ is plotted as green profile with axis on the right. The same causality as discussed before is seen in more detail here. Before RMP application no indication for a strike line striation is seen. During the ideal screening phase, a small modulation is apparent and the location of the maxima agrees with the location of the hit points of the separatrix lobes which guide field line penetrating in the vacuum approach as deep as $\Delta \Psi_{\mathrm{N}} \sim 0.1$ into the plasma. However, the modulation amplitude in the $\operatorname{CII}\left(\Delta s_{\text {wall }}\right)$ profile is small in this stage and the actual $\mathrm{C}_{\mathrm{II}}$ emission is blurred. This indicates that no direct flow to the target happens but that the perturbed lobe structure is filled by a radial diffusion of particles. This changes considerably when the plasma response decayed. Now the striation is visible in high contrast and a clear striation is

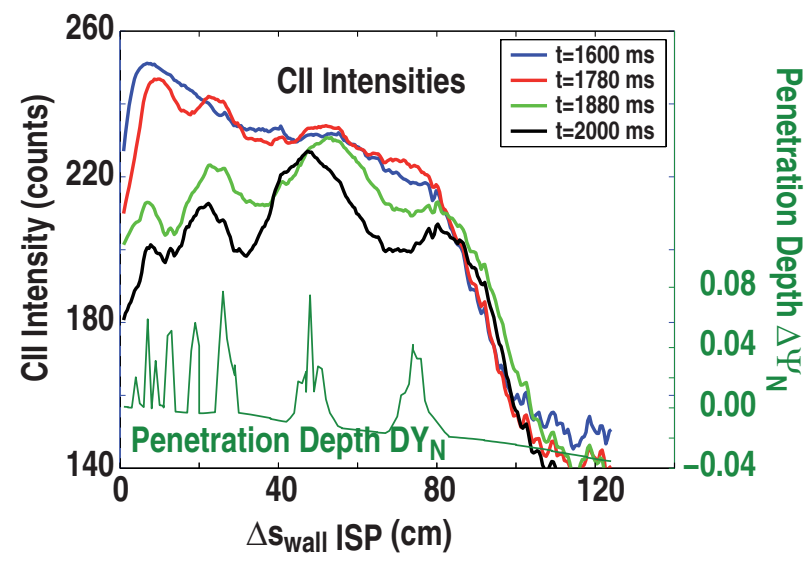

Figure 7. $\mathrm{C}$ II emission profiles at the inner divertor leg during decay of the plasma response.

measured. The maxima of the $\mathrm{CII}\left(\Delta s_{\text {wall }}\right)$ profile agree with the location of the separatrix lobes confirming that for this penetrated state, the vacuum modelled magnetic footprint agrees with the measured location of the $\mathrm{C} \operatorname{II}\left(\Delta s_{\text {wall }}\right)$ striated pattern. In an attached divertor state, the $\mathrm{C}$ II $\left(\Delta s_{\text {wall }}\right)$ emission can be taken as a measure for the incoming ion flow and-as it is dominated by the carbon source-for the localization of the sputtered carbon at the target surface. The observation that the total intensity decreases when the penetrated state is reached suggests a reduction of the carbon source potentially because of a reduced target heat flux or a reduction of the divertor density. Both effects are compatible with formation of a 3D separatrix structure inducing a wider heat flux distribution or a wider distribution of recycling due to the helical footprint formed. Hence, these measurements show that the separatrix manifolds are decomposed and a 3D helical separatrix structure with helical particle fluxes onto the divertor plates are formed as soon as the plasma response decayed. This shows that at this point the good flux surfaces close to the separatrix are broken up and direct parallel transport channels to the wall from inside of the unperturbed separatrix are formed. However, the radial depth of this domain of open, perturbed field lines cannot yet be specified.

\section{Conclusion and discussion}

The measurements presented in this paper show that a LSN L-mode plasma is capable of shielding the external RMP field by an ideal resonant screening response with an $n=3$ plasma response field. As soon as this response field decays, we observe in a tangential view as well as in a direct inner divertor target plate view a 3D deformation of the plasma boundary with a strike line splitting in fair agreement with the vacuum magnetic footprint modelling. As this is one of the most reliable features during ELM suppression this first time observation in poloidally diverted L-mode plasmas provides essential information to understand the link between the plasma response and the observation of strike line striation. The fact that already during the response period a small modulation of $\mathrm{C} I I\left(\Delta s_{\text {wall }}\right)$ is detected suggests that a small field remains at the separatrix capable of decomposing the separatrix manifolds. When this plasma response decayedjudging from the comparison at the divertor surface- the lobe 
location matches the vacuum field line tracing result. This shows that the separatrix lobes exist and that they overlap inside of the unperturbed separatrix. Hence, it proves that the radial domain of the overlapping separatrix lobes-typical $2 \%-3 \%$ in normalized toroidal flux - is opened to the wall and stochastized. As the small oscillations in the carbon emission for the screened case are located at the same radial location, the overlap and hence stochastic region domain can be estimated with a similar penetration depth. This interpretations means that the separatrix deformation is similar but the inward extension of the stochastic layer is different for the penetrated and the screened case. In order to determine the inward extension of the stochastic layer suggested by the transport enhancement measured in the particle confinement and the rotation spin up advanced models are required. Simulations for the field topology including the ideal plasma response and-for reference-also from ideal or resistive approaches [37,38] or drift kinetic attempts [39] need to be performed and compared to these measurements. This will allow benchmarking of the edge perturbation against a given level of internal resonant field penetration or screening and enables the setting of realistic margins for the penetration depth of the perturbed stochastic boundary layer.

\section{Acknowledgments}

This work was supported by the US Department of Energy under DE-FG03-97ER54415, DE-AC52-07NA27344, DE-FC02-04ER57698, DE-FG02-07ER54917, DE-AC05DOOR22725 and DE-FG02-89ER53297.

\section{References}

[1] Wagner F. et al 1982 Phys. Rev. Lett. 491408

[2] Loarte A. et al 2003 Plasma Phys. Control. Fusion 451549
[3] Evans T.E. et al 2006 Phys. Rev. Lett. 92235003

[4] Evans T.E. et al 2006 Nature Phys. 2419

[5] Evans T.E. et al 2008 Nucl. Fusion 48024002

[6] Suttrop W. et al 2011 Phys. Rev. Lett. 106225004

[7] Liang Y. et al 2010 Nucl. Fusion 50025013

[8] Jeon Y.M. et al 2012 Phys. Rev. Lett. 109035004

[9] Nardon E. et al 2009 Plasma Phys. Control. Fusion 51124010

[10] Kirk A. et al 2013 Nucl. Fusion 53043007

[11] Canik J.M. et al 2010 Nucl. Fusion 50034012

[12] Fenstermacher M.E. et al 2008 Phys. Plasmas 15056122

[13] Schmitz O. et al 2009 Phys. Rev. Lett. 103165005

[14] Schmitz O. et al 2012 Nucl. Fusion 52043005

[15] Stoschus H. et al 2013 Nucl. Fusion 53012001

[16] Evans T.E. et al 2005 J. Phys.: Conf. Ser. 7174

[17] Schmitz O. et al 2008 Plasma Phys. Control. Fusion 50124029

[18] Jakubowski M.W. et al 2009 Nucl. Fusion 49095013

[19] Evans T.E. 2008 Chaos, Complexity and Transport (Singapore: World Scientific)

[20] Wingen A. et al 2007 Phys. Plasmas 14042502

[21] Shafer M.W. et al 2012 Nucl. Fusion 52122001

[22] Kirk A. et al 2012 Phys. Rev. Lett. 108255003

[23] Ahn J.W., Canik J.M., Soukhanovskii V.A., Maingi R. and Battaglia D.J. 2010 Nucl. Fusion 50045010

[24] Nardon E. et al 2011 J. Nucl. Mater. 363-395 1071

[25] Srait E.J. et al 2005 Fusion Sci. Technol. 48864

[26] Liu Y.Q. et al 2000 Phys. Plasmas 73681

[27] Lanctot M.J. et al 2010 Phys. Plasmas 17030701

[28] Fitzpatrick R. 1993 Nucl. Fusion 331049

[29] Mordijck S. et al 2012 Phys. Plasmas 19056503

[30] Ghendrih Ph. et al 2002 Nucl. Fusion 421221

[31] Schmitz O. et al 2009 J. Nucl. Mater. 390-391 299

[32] Watkins J.G., Evans T.E., Lasnier C.J., Moyer R.A. and Rudakov D.L. 2007 J. Nucl. Mater. 363-365 708

[33] Unterberg B. et al 2007 J. Nucl. Mater. 363-365 698

[34] Wingen A. and Spatschek K.H. 2008 Phys. Plasmas 15052305

[35] Coenen J.W. et al 2011 Nucl. Fusion 51063030

[36] Fenstermacher M.E. et al 1997 Rev. Sci. Instrum. 6874

[37] Ferraro N.W., Jardin S.C. and Snyder P.B. 2010 Phys. Plasmas 17102508

[38] Izzo V.A. and Joseph I. 2008 Nucl. Fusion 48115004

[39] Reiser D. and Chandra D. 2009 Phys. Plasmas 16042317 